\title{
GENERALIZATIONS OF A PROPERTY OF TCHEBYCHEFF POLYNOMIALS
}

\author{
THOMAS L. SHERMAN
}

The purpose of this paper is to generalize a well-known property of polynomials to functions which are solutions of linear differential equations of the form $L_{n} y=\sum_{i=0}^{n} p_{i}(x) y^{(i)}=0$, where $p_{n}(x) \neq 0$ and all the coefficients are continuous. In particular, the problem to be generalized is that of finding a polynomial of degree $n$ with leading coefficient 1 whose maximum absolute value deviates least from zero in the interval $-1 \leqq x \leqq 1$ (see for example Courant and Hilbert [1, pp. 88-89]).

First we need:

Definition. The first conjugate point $\eta_{1}(a)$ of the point $a$ is the smallest number $b>a$ such that there exists a nontrivial solution of $L_{n} y=0$ which vanishes at $a$ and has $n$ zeros, counting multiplications, in $[a, b]$.

We can now state the main result of this paper.

ThEOREM. Let $L_{n+k} y=\sum_{i=0}^{n+k} p_{n+k, i}(x) y^{(i)}=0(k=0,1)$ be a pair of linear differential equations, where $p_{n+k, n+k}(x) \neq 0(k=0,1), n \geqq 1$ and all the coefficients are continuous on $[a, b]$ and, $b \leqq \eta_{1}(a)$ for $k=0,1$ (if $\eta_{1}(a)$ does not exist, $b$ may be chosen arbitrarily). Suppose that if $z(x)$ is a solution of $L_{n} y=0$, then it is also a solution of $L_{n+1} y=0$. Let $\left\{\phi_{i}(x)\right\}$ $(i=1, \cdots, n+1)$ be a fundamental set of solutions of $L_{n+1} y=0$, where $\left\{\phi_{i}(x)\right\}(i=1, \cdots, n)$ is a fundamental set of solutions of $L_{n} y=0$. Thenthesolution $\phi(x)$ of $L_{n+1} y=0$, such that $\phi(x)=\phi_{n+1}(x)+\sum_{i=1}^{n} a_{i} \phi_{i}(x)$ and such that $\max _{x \in[a, b]}|\phi(x)|$ is minimal, is that solution $\phi(x)$ satisfying

(i) $\phi(x)$ has $n$ distinct zeros in $(a, b)$.

(ii) Let the zeros in (i) be located at $x_{1}<x_{2}<\cdots<x_{n}$. Let the points at which $\phi(x)$ attains its extreme values in $\left[a, x_{1}\right],\left[x_{1}, x_{2}\right], \cdots$, $\left[x_{n-1}, x_{n}\right],\left[x_{n}, b\right]$ be denoted respectively by $c_{1}, c_{2}, \cdots, c_{n}, c_{n+1}$. Then $\left|\phi\left(c_{1}\right)\right|=\left|\phi\left(c_{2}\right)\right|=\cdots=\left|\phi\left(c_{n+1}\right)\right|$.

Proof. First it must be shown that such a solution $\phi(x)$ of $L_{n+1} y=0$ exists. We can always assign $n$ zeros in $(a, b)$ in an arbitrary manner $[2$, Theorem 3]. We need first to show that these points may be adjusted such that the relative values $\left|\phi\left(c_{i}\right)\right|(i=1, \cdots, n+1)$ may be adjusted at will. Denote by $\|y\|$ the norm $\sum_{i=0}^{n} \max _{x \in[a, b]}\left|y^{(i)}(x)\right|$.

Received by the editors January 1, 1967. 
Let $S=\left\{y \mid y\right.$ is a solution of $L_{n+1} y=0$ with $n$ distinct zeros on $(a, b)$ and $\|y\|=1\}$. First fix $x_{2}, x_{3}, \cdots, x_{n}$ for $y \in S$ and let $x_{1}$ approach $a$. Then $y\left(c_{1}\right)$ approaches zero since $\|y\|=1$. Similarly $y\left(c_{i}\right) \rightarrow 0$ as $x_{i}-x_{i-1} \rightarrow 0(i=2,3 \cdots, n)$, but $y\left(c_{j}\right) \rightarrow 0(i \neq j)$ since $\eta_{1}(a) \geqq b$ (for $\left.L_{n+1} y=0\right)$ implies that no solution has $n+1$ zeros on $[a, b)$ (or $\left.(a, b]\right)$, by [2]. Thus by appropriate choice of $x_{1}, \cdots, x_{n}$ we can adjust the relative values of $\left|y\left(c_{i}\right)\right|$.

We shall now establish the existence of the solution $\phi(x)$ of the theorem. By choosing $x_{1}$ sufficiently near $a$ we can choose a solution $y(x) \in S$ such that $\left|y\left(c_{1}\right)\right| \leqq\left|y\left(c_{i}\right)\right|, i>1$. Now let $x_{2} \rightarrow x_{1}$ until $\left|y\left(c_{1}\right)\right|=\left|y\left(c_{2}\right)\right|$, then by choosing both $x_{1}$ and $x_{2}$ sufficiently close to $a$ we find $\left|y\left(c_{1}\right)\right|=\left|y\left(c_{2}\right)\right| \leqq\left|y\left(c_{i}\right)\right|, i>2$. Continuing in this manner we find a solution $y(x)=\Psi_{1}(x) \in S$ such that $\left|\Psi_{1}\left(c_{1}\right)\right|=\left|\Psi_{1}\left(c_{2}\right)\right|$ $=\cdots=\left|\Psi_{1}\left(c_{n}\right)\right| \leqq\left|\Psi\left(c_{n+1}\right)\right|$. We now shall vary the points $x_{1}$, $\cdots, x_{n}$ as determined by $\Psi_{1}(x)=y(x)$. Let $x_{n} \rightarrow b$ until $\left|y\left(c_{n+1}\right)\right|$ $=\left|y\left(c_{j}\right)\right|$ for some $j<n+1$. Let $c_{j_{1}}<c_{j_{2}}<\cdots<c_{j_{\text {s }}}$ be the values such that $\left|y\left(c_{n+1}\right)\right|=\left|y\left(c_{j_{1}}\right)\right|=\left|y\left(c_{j_{2}}\right)\right|=\cdots=\left|y\left(c_{j_{s}}\right)\right|$. If $s=n$ we are of course done, so we shall suppose $s<n$. Let $k$ be the smallest integer for which the sequences $j_{1}, j_{2}, \cdots, j_{k}$ and $1,2, \cdots, k$ do not coincide. For $j_{i}<k$ let $x_{j_{i}} \rightarrow x_{j_{i}-1}$ (and if $j_{1}=1$ let $x_{j_{1}} \rightarrow a$ ), for $j_{i}>k$ let $x_{j_{i}-1} \rightarrow x_{j_{i}}$ and $x_{n} \rightarrow b$ (that is the zero points to the left of $x_{k}$ are moved to the left and the zero poin ts to the right of $x_{k}$ are moved to the right) until $\left|y\left(c_{n+1}\right)\right|=\left|y\left(c_{j_{i}}\right)\right|=\cdots=\left|y\left(c_{j_{s}}\right)\right|=\left|y\left(c_{m}\right)\right|$ for some $m$ $\notin\left\{j_{1}, j_{2}, \cdots, j_{s}, n+1\right\}$. Continuing in this manner we can find a solution $y(x)=\Psi_{2}(x) \in S$ such that $\left|\Psi_{2}\left(c_{n+1}\right)\right|=\left|\Psi_{2}\left(c_{i}\right)\right| \geqq\left|\Psi_{2}\left(c_{j}\right)\right|$ for some $j<n+1$ and all $i \neq j, i<n+1$. In a manner similar to that used to find $\Psi_{1}(x)$ we can find a solution $\Psi_{3}(x) \in S$ such that $\left|\Psi_{3}\left(c_{n+1}\right)\right|$ $=\left|\Psi_{3}\left(c_{i}\right)\right| \leqq\left|\Psi_{3}\left(c_{j}\right)\right|$ for all $i \neq j, i<n+1$. Comparing $\Psi_{3}(x)$ and $\Psi_{2}(x)$ we conclude, from the continuous dependence of solutions on $x_{1}, x_{2}$, $\cdots, x_{n}$, that the solution $\phi(x)$ of the theorem exists. Further, since $\eta_{1}(a) \geqq b$ (for $L_{n} y=0$ ) implies there is no solution of $L_{n} y=0$ with $n$ zeros in $(a, b)$, we conclude $\phi(x)$ is a solution of $L_{n+1} y=0$ but not of $L_{n} y=0$. Hence, after multiplying $\phi(x)$ by a nonzero constant if necessary, we may write $\phi(x)=\phi_{n+1}(x)+\sum_{i=1}^{n} a_{i} \phi_{i}(x)$ (we drop the requirement here that the solution be a member of $S$ ).

We shall now show that this solution $\phi(x)$ has the desired extremal property. Suppose it does not, then there is a solution $z(x)=\phi_{n+1}(x)$ $+\sum_{i=1}^{n} b_{i} \phi_{i}(x) \quad$ such that $\max _{x \in[a, b]}|z(x)|<\max _{x \in[a, b]}|\phi(x)|$ $=\left|\phi\left(c_{i}\right)\right| \quad(i=1,2, \cdots, n+1)$. We shall for definiteness assume $\phi(a)>0$. Then $\phi\left(c_{1}\right)-z\left(c_{1}\right)>0, \phi\left(c_{2}\right)-z\left(c_{2}\right)<0, \cdots$. Hence $\phi(x)$ $-z(x)=\sum_{i=1}^{n}\left(a_{i}-b_{i}\right) \phi_{i}(x)$ vanishes at least once in $\left(c_{1}, c_{2}\right),\left(c_{2}, c_{3}\right)$, 
$\cdots,\left(c_{n}, c_{n+1}\right)$ and hence has $n$ zeros in $(a, b)$. However, $\phi(x)-z(x)$ is then a solution of $L_{n} y=0$ with $n$ zeros in $(a, b)$ which is impossible since $\eta_{1}(a) \geqq b$, by [2].

Corollary. Let $L_{n+k} y=\sum_{i=1}^{n+k} p_{n+k, i}(x) y^{(i)}=0 \quad(k=0,2)$ be a pair of linear differential equations, where $p_{n+k, n+k}(x) \neq 0(k=0,2), n \geqq 1$ and all the coefficients are continuous on $[a, b]$ and $b \leqq \eta_{1}(a)$ for $k=0,2$ (if $\eta_{1}(a)$ does not exist, $b$ may be chosen arbitrarily). Suppose that if $z(x)$ is a solution of $L_{n} y=0$, then it is also a solution of $L_{n+2} y=0$. Let $\left\{\phi_{i}(x)\right\}(i=1, \cdots, n)$ be a fundamental set of solutions of $L_{n} y=0$. Let $\phi_{n+2}(x)$ be a solution of $L_{n+2} y=0$ which has $n+1$ distinct zeros in $(a, b)$ located at $x_{1}<x_{2}<\cdots<x_{n+1}$ such that $\max _{x \in\left[a, x_{1}\right]}\left|\phi_{n+2}(x)\right|$ $=\max _{x \in\left[x_{1}, x_{2}\right]}\left|\phi_{n+2}(x)\right|=\cdots=\max _{x \in\left[x_{n+1, b}\right]}\left|\phi_{n+2}(x)\right|$ (i.e. $\phi_{n+2}(x)$ is the solution $\phi(x)$ of the theorem). Let the points at which their extreme values are attained be denoted by $c_{1}, c_{2}, \cdots c_{n+2}$ respectively. Let $\phi_{n+1}(x)$ be a solution of $L_{n+2} y=0$ which satisfies the conditions $\phi\left(c_{i}\right)=0$ for $i=1,2, \cdots, n+1$. Then the solution $\phi(x)$ of $L_{n+2} y=0$, such that $\phi(x)=\phi_{n+2}(x)+\sum_{i=2}^{n+1} a_{i} \phi_{i}(x)$ and such that $\max _{x \in[a, b]}|\phi(x)|$ is minimal, is $\phi(x)=\phi_{n+2}(x)$.

Proof. We first note that $\phi_{n+1}(x)$ is not a solution of $L_{n} y=0$ since it has $n+1$ zeros on $[a, b)$, hence it is independent of $\phi_{i}(x)(i=1,2$, $\cdots, n)$. Further it is independent of $\phi_{n+2}(x)$ since the zeros are different. Suppose now $\phi_{n+2}$ does not have the desired extremal property. Then there is a solution $z(x)=\phi_{n+2}(x)+\sum_{i=1}^{n+1} b_{i} \phi_{i}(x)$ such that $\max _{x \in[a, b]}|z(x)|<\max _{x \in[a, b]}\left|\phi_{n+2}(x)\right|$. We shall assume $\phi_{n+2}(a)>0$. In particular $\phi_{n+2}\left(c_{1}\right)-z\left(c_{1}\right)>0, \phi_{n+2}\left(c_{2}\right)-z\left(c_{2}\right)<0, \cdots$, but, since $\phi_{n+1}\left(c_{i}\right)=0 \quad(i=1, \cdots, n+1), \phi_{n+2}\left(c_{i}\right)-\left[z\left(c_{i}\right)-b_{n+1} \phi_{n+1}\left(c_{i}\right)\right]$ has these same properties for $i=1, \cdots, n+1$. Hence $\phi_{n+2}(x)$ $-\left[z(x)-b_{n+1} \phi_{n+1}(x)\right]=-\sum_{i=1}^{n} b_{i} \phi_{i}(x)$ vanishes at least once in $\left(c_{1}, c_{2}\right),\left(c_{2}, c_{3}\right), \cdots,\left(c_{n}, c_{n+1}\right)$ and hence has $n$ zeros in $(a, b)$. But, since $\eta_{1}(a) \geqq b$ for $L_{n} y=0$ and $-\sum_{i=0}^{n} b_{i} \phi_{i}(x)$ is a solution of $L_{n} y$ $=0$, we conclude that $b_{i}=0 \quad(i=1, \cdots, n)$. Hence $z(x)$ $=\phi_{n+2}(x)+b_{n+1} \phi_{n+1}(x)$. However $z\left(c_{1}\right)=\phi_{n+2}\left(c_{1}\right)$, contradicting the assumption that $\phi_{n+2}\left(c_{1}\right)=\max _{x \in[a, b]}\left|\phi_{n+2}(x)\right|>\max _{x \in[a, b]}|z(x)|$. Hence $\phi_{n+2}(x)$ is the desired solution.

ExAmpLES. (1) Let $L_{n+k} y=y^{(n+k)}=0(k=0,1), a=-1, b=1$, and $\phi_{i}(x)=x^{i-1}(i=1,2, \cdots, n+1)$. In this case the solutions in question are polynomials of degree $n$ with leading coefficient one. The function $\phi(x)$ of the theorem, as in well known, is the Tchebycheff polynomial $T_{n}(x)=\left(\frac{1}{2}\right)^{n-1} \cos \left[n \cos ^{-1} x\right]$.

(2) Let $n=2 m+1$ and let 


$$
\begin{aligned}
L_{n+k} y=y^{(n+k)} & +\sum_{i=1}^{m+k / 2} i^{2} y^{(n+k-2)}+\sum_{j=2}^{m+k / 2} \sum_{i<j} i^{2} j^{2} y^{(n+k-4)} \\
& +\sum_{m=3}^{m+k / 2} \sum_{j<m} \sum_{i<j} i^{2} j^{2} m^{2} y^{(n+k-6)}+\cdots \\
& +\left(1^{2} \cdot 2^{2} \ldots \ldots(m+k / 2)^{2}\right) y^{\prime}=0,
\end{aligned}
$$

where $(k=0,2), a=0, b=2 \pi$ and $\phi_{1}(x)=1, \phi_{2}(x)=\sin x, \phi_{3}(x)=\cos x$, . . , $\phi_{n}(x)=\cos m x, \phi_{n+1}(x)=\sin (m+1) x, \phi_{n+2}(x)=\cos (m+1) x$. Thus we are considering the usual Fourier series partial sum of order $m+1$ with leading coefficient one. The function $\phi_{n+2}(x)=\cos (m+1) x$ is the function of the corollary.

\section{BIBLIOGRAPHY}

1. R. Courant and D. Hilbert, Methods of mathematical physics, Vol. 1, Interscience, New York, 1953.

2. T. L. Sherman, Properties of solutions of Nth order linear differential equations, Pacific J. Math. 15 (1965), 1045-1060.

Arizona State University 